\title{
A Phos-Tag-Based Fluorescence Quenching System for Activity Assay and Inhibitor Screening for Alkaline Phosphatase
}

\author{
Emiko Kinoshita-Kikuta1, Hiromasa Kurosaki², Natsumi Kunisada1, Eiji Kinoshita ${ }^{{ }^{*}}$, \\ Tohru Koike ${ }^{1^{*}}$ \\ ${ }^{1}$ Department of Functional Molecular Science, Institute of Biomedical \& Health Sciences, Hiroshima University, \\ Hiroshima, Japan \\ ${ }^{2}$ Department of Structure-Function Physical Chemistry, Graduate School of Pharmaceutical Sciences, \\ Kumamoto University, Kumamoto, Japan \\ Email: "kinoeiii@hiroshima-u.ac.jp, ${ }^{*}$ tkoike@hiroshima-u.ac.jp
}

Received 23 June 2014; revised 12 August 2014; accepted 28 August 2014

Copyright @ 2014 by authors and Scientific Research Publishing Inc.

This work is licensed under the Creative Commons Attribution International License (CC BY).

http://creativecommons.org/licenses/by/4.0/

(c) $\stackrel{\text { (i) }}{\mathrm{EY}}$ Open Access

\begin{abstract}
Fluorescence resonance energy transfer (FRET) is a distance-dependent interaction between the electronic excited states of two dye molecules. Here we introduce a novel FRET-based fluorescence quenching system for assaying the activity of alkaline phosphatase (AP) by using a phosphate-binding tag molecule, Phos-tag \{1,3-bis[bis(pyridine-2-ylmethyl)amino]propan-2-olato dizinc(II) complex $\}$, attached to a nonfluorescent 4-\{[4-(dimethylamino)phenyl]diazenyl\}benzoyl (Dabcyl: $\lambda_{\max } 475 \mathrm{~nm}$ ) dye group. The fluorogenic biomolecule riboflavin 5'-phosphate (FMN: $\lambda_{\text {em }}$ $525 \mathrm{~nm}$ ) was used as an AP substrate. The Dabcyl-labeled Phos-tag specifically captured FMN to form a stable 1:1 complex, resulting in efficient fluorescence quenching. The quenching efficiency was more than $95 \%$ for a mixture of $12 \mu \mathrm{M}$ FMN and 13.5 $\mu \mathrm{M}$ Dabcyl-labeled Phos-tag in aqueous solution at $\mathrm{pH} 7.4$ and $25^{\circ} \mathrm{C}$. When FMN was dephosphorylated with AP, riboflavin was released into the solution and fluorescence from the flavin moiety appeared. By using this quenching system, we succeeded in detecting time- and dose-dependent dephosphorylation of FMN by AP under near-physiological conditions.
\end{abstract}

\section{Keywords}

Fluorescence Resonance Energy Transfer, Enzyme Assays, Enzyme Inhibitors, Phos-Tag, Alkaline Phosphatase

\footnotetext{
${ }^{*}$ Corresponding authors.
} 


\section{Introduction}

Alkaline phosphatases (APs, EC 3.1.3.1) are found in many organisms, from bacteria to mammals [1]. The enzymes catalyze hydrolyses of various phosphoric monoesters and transphosphorylations of phosphorylated species to other molecules [2]. As one of the most commonly assayed enzymes, serum AP is widely used as a clinical indicator for several diseases, including liver dysfunction, several kinds of cancer, and diabetes [3] [4]. Several assays for the activity of AP have been reported; these generally involve colorimetric, chromatographic, radioactive, or electrochemical approaches. Unlike many of these assays, fluorescence-based methods do not necessarily require the use of radioactive or immunoactive labels, and they are therefore attractive as convenient and reliable procedures for the analysis of AP activity [5]-[7].

We previously reported that the dinuclear metal complex 1,3-bis[bis(pyridin-2-ylmethyl)amino]propan-2olato dizinc(II) (Phos-tag) acted as a phosphate-binding tag molecule under near-physiological conditions in aqueous solution at neutral $\mathrm{pH}$ values [8]. As a result, a number of original analytical methods that use various Phos-tag derivatives have been developed for research on the phosphoproteome [9]-[12]. In 2009, the phosphate-capturing ability of an aminocoumarin-attached Phos-tag molecule was utilized in the development of a fluorescence resonance energy transfer (FRET) system for the analysis of the dephosphorylation of a fluorescein-labeled phosphopeptide substrate by bovine intestinal AP [13]. The assay is based on the principle that the Phos-tag derivative captures the fluorogenic phosphopeptide in preference to its nonphosphorylated counterpart. The formation of a 1:1 complex between the Phos-tag moiety and the phosphopeptide brings the donor near the acceptor, resulting in FRET with an efficiency that varies from $47 \%$ to $86 \%$, depending on the type of peptide sequence. Furthermore, we applied a similar FRET system to an examination of the reverse reaction, phosphorylation, of a fluorescein-labeled peptide substrate by a certain kinase [14].

To date, several fluorescence-quenching systems that use a fluorophore-quencher (donor-acceptor) pair have been developed for the analysis of biomolecules such as nucleic acids or peptides [15] [16]. If the donor and acceptor molecules approach one another closely, the fluorescence from the donor group is efficiently reduced by the acceptor. In the case, the acceptor is referred to as a "dark quencher" or "black-hole quencher". One of the most commonly used quenchers is the 4-([4-(dimethylamino)phenyl]diazenyl)benzoyl group (Dabcyl), which has a strong absorption in the visible region of the spectrum [17]. In this study, we introduce a novel quencher, Dabcyl-labeled Phos-tag (Dabcyl-Phos-tag, $\lambda_{\max }=475 \mathrm{~nm}$ ), which preferentially captures the fluorogenic APsubstrate riboflavin 5'-phosphate \{1-deoxy-1-(7,8-dimethyl-2,4-dioxo-3,4-dihydrobenzo[g]pteridin-10(2H)-yl)5-O-phosphono-D-ribitol\}, also known as flavin mononucleotide (FMN) [18], at micromolar concentrations. The emission spectrum of FMN ( $\lambda_{\mathrm{em}}=525 \mathrm{~nm}$ ) efficiently overlaps the absorption spectrum of the Dabcyl group.

As a first practical example of the use of Dabcyl-Phos-tag, we demonstrate the detection of the time-dependent dephosphorylation of FMN by AP under near-physiological conditions. Furthermore, dose-dependent inhibitions of AP activity by the well-known inhibitors vanadate [19] [20] and (6S)-6-phenyl-2,3,5,6-tetrahydroimidazo[2,1-b][1,3]thiazole (levamisole) [21] [22] were also examined by using the Phos-tag-based fluorescence quenching system.

\section{Materials and Methods}

\subsection{Materials}

1-[(4-\{[4-(Dimethylamino)phenyl]diazenyl\}benzoyl)oxy]pyrrolidine-2,5-dione (Dabcyl NHS ester) was purchased from Tokyo Chemical Industry Co., Ltd. (Tokyo, Japan). Disodium 2,2',2', 2,'”-(1,2-ethanediyldiammonio) tetraacetate (disodium EDTA) and 2-[4-(2-hydroxyethyl)piperazin-1-yl]ethane-1-sulfonic acid (Hepes) were purchased from Nacalai Tesque, Inc. (Kyoto, Japan). Sodium orthovanadate $\left(\mathrm{Na}_{3} \mathrm{VO}_{4}\right)$ was purchased from Calbiochem (La Jolla, CA, USA). Levamisole was purchased from Sigma-Aldrich (St. Louis, MO, USA).FMN monosodium salt (purity >95\%) and riboflavin, both of which have the same $\lambda_{\max }$ value of $445 \mathrm{~nm}(\varepsilon=1.25 \times$ $10^{4} \mathrm{M}^{-1} \cdot \mathrm{cm}^{-1}$ ) in $\mathrm{H}_{2} \mathrm{O}$ [18], were purchased from Wako Pure Chemical Industries, Ltd. (Osaka, Japan): FMN was used after purification ( $>99 \%$ ) by column chromatography on ODS silica gel, Cosmosil $140 \mathrm{C}_{18}$-OPN (Nacalai Tesque). Bovine intestinal mucosa AP Type VII-S and bovine kidney AP were purchased from Sigma-Aldrich. One unit of the phosphatase hydrolyzed $1 \mu \mathrm{mol}$ of 4-nitrophenylphosphate per minute at $\mathrm{pH} 9.8$ and $37^{\circ} \mathrm{C}$.

All aqueous solutions were prepared by using distilled water. All chemical reagents and solvents were of the highest commercial quality and were used without further purification. TLC was performed on TLC silica gel 
plates $60 \mathrm{NH}_{2} \mathrm{~F}_{254 \mathrm{~s}}$ (No. 5533; Merck, Darmstadt, Germany) and silica gel column chromatography was performed on silica gel NH-DM 1020 (Fuji Silysia Chemical, Ltd.; Kasugai, Japan).

\subsection{Apparatus}

UV and visible spectra were recorded on a V-630 spectrophotometer (JASCO Corp.; Tokyo, Japan) at $25.0^{\circ} \mathrm{C} \pm$ $0.1^{\circ} \mathrm{C}$. Fluorescence spectra were obtained with an F-2500 fluorescence spectrophotometer (Hitachi High-Technologies Corp.; Tokyo, Japan) equipped with a 1 -cm quartz cell at $25.0^{\circ} \mathrm{C} \pm 0.1^{\circ} \mathrm{C}$. IR spectra were recorded on an FT-710 IR spectrophotometer (Horiba Ltd.; Kyoto, Japan) at room temperature. ${ }^{1} \mathrm{H}(500 \mathrm{MHz})$ and ${ }^{13} \mathrm{C}(125$ MHz) NMR spectra were recorded on a JEOL LA500 spectrometer with a field-gradient unit (JEOL Ltd.; Tokyo, Japan) at $25^{\circ} \mathrm{C}$. Tetramethylsilane (in $\mathrm{CDCl}_{3}$ ) was used as an internal reference for the NMR measurements. The ${ }^{1} \mathrm{H}$ and ${ }^{13} \mathrm{C}$ signals were assigned by means of $1 \mathrm{D}$ and 2D ( ${ }^{1} \mathrm{H}$ COSY, HMQC and HMBC) NMR experiments. The $\mathrm{pH}$ measurements were conducted with an F-53 pH meter (Horiba Ltd.; Kyoto, Japan) equipped with a combination $\mathrm{pH}$ electrode (Horiba-6378) calibrated by using $\mathrm{pH}$ standard buffers $(\mathrm{pH} 4.01$ and 6.86$)$ at $25^{\circ} \mathrm{C}$. HPLC was performed by using an HPLC system (JASCO Corp.) consisting of a column oven (CO-2060), a UV detector (UV-2070), a degasser (DG-2080-53), and two pumps (PU-2080 plus). The flow rate was $1.0 \mathrm{ml} / \mathrm{min}$ and the column temperature was $40^{\circ} \mathrm{C}$. Purities of FMN and riboflavin were determined by HPLC with a reverse-phase column (Shiseido CAPCELL PAK C18 UG80, $4.6 \times 150 \mathrm{~mm}$; Yokohama, Japan) eluted by a 7:3 mixture of $10 \mathrm{mM}$ phosphate buffer (pH 5.5) and methanol. The retention times for FMN and riboflavin were $2.8 \mathrm{~min}$ and $4.2 \mathrm{~min}$, respectively.

\subsection{Synthesis of the Dabcyl-Labeled Phos-Tag Ligand}

An amino-pendent Phos-tag ligand attached to a hydrophilic 15-atom spacer, $N$-[3-(2-[2-(3-aminopropoxy) ethoxy]ethoxy)propyl]-6-([(3-[bis(pyridin-2-ylmethyl)amino]-2-hydroxypropyl)(pyridin-2-ylmethyl)amino]methyl)nicotinamide, was synthesized as described previously [12]. A solution of Dabcyl NHS ester (185 mg, 0.50 mmol) in $\mathrm{CHCl}_{3}(5 \mathrm{ml})$ was added to a solution of the amino-pendent Phos-tag ligand $(0.35 \mathrm{~g}, 0.50 \mathrm{mmol})$ in $\mathrm{CHCl}_{3}(5 \mathrm{ml})$ at room temperature and allowed to react. The mixture was then stirred gently for $24 \mathrm{~h}$ at room temperature. $\mathrm{CHCl}_{3}(50 \mathrm{ml})$ was added, and the solution was washed with distilled water $(2 \times 50 \mathrm{~mL})$. The $\mathrm{CHCl}_{3}$ layer was separated, dried $\left(\mathrm{Na}_{2} \mathrm{SO}_{4}\right)$, and concentrated. The residue was purified by column chromatography [silica gel (NH-DM1020), $\mathrm{CHCl}_{3}-\mathrm{MeOH}$ (50:1)] to give the Dabcyl-labeled Phos-tag ligand, 6- $\{[\{3-[$ bis(pyridin2-ylmethyl)amino]-2-hydroxypropyl $\}$ (pyridin-2-ylmethyl)amino]methyl $\}-N$-[15-(4- $\{(E)$-[4-(dimethylamino)phenyl]diazenyl \}phenyl)-15-oxo-4,7,10-trioxa-14-azapentadec-1-yl]nicotinamide, as a dark-red viscous solid; yield: $0.40 \mathrm{~g}(0.42 \mathrm{mmol}, 84 \%)$. TLC $\left(\mathrm{CHCl}_{3}-\mathrm{MeOH}=25: 1\right): R_{f}=0.59$ (one spot). IR (KCl): 3320 (br), 2950, 2866, 1643, 1600, 1547, 1521, 1478, 1422, 1365, 1311, 1251, 1138, 946, 861, 823, 761, $664 \mathrm{~cm}^{-1} .{ }^{1} \mathrm{H}$ NMR (500 MHz, $\left.\mathrm{CDCl}_{3}\right): \delta=1.80-1.89\left(\mathrm{~m}, 4 \mathrm{H}, \mathrm{OCCH}_{2} \mathrm{CN}\right), 2.23(\mathrm{br} \mathrm{s}, 1 \mathrm{H}, \mathrm{OH}), 2.54-2.69\left(\mathrm{~m}, 4 \mathrm{H}, \mathrm{NCH}_{2} \mathrm{CCH}_{2} \mathrm{~N}\right), 3.09(\mathrm{~s}$, $\left.6 \mathrm{H}, \mathrm{CH}_{3}\right), 3.49$ - 3.63 (m, 16H, $\left.\mathrm{CONCH}_{2} \mathrm{C}, \mathrm{OCH}_{2} \mathrm{C}\right), 3.80$ - 3.91 (m, 8H, $\left.\mathrm{PyCH}_{2}\right), 3.91$ - 3.98 (m, 1H, NCCHCN), 6.74 (d, 2H, $J=9.2 \mathrm{~Hz}, \mathrm{ArH}), 7.08-7.12(\mathrm{~m}, 3 \mathrm{H}, \mathrm{PyH}), 7.31-7.36$ (m, 4H, PyH, CONH), 7.38 (br s, $1 \mathrm{H}$, CONH), 7.44 (d, $J=7.9 \mathrm{~Hz}, 1 \mathrm{H}, \mathrm{PyH}), 7.53-7.59$ (m, 3H, PyH), 7.82 (d, $J=8.5 \mathrm{~Hz}, 2 \mathrm{H}, \mathrm{ArH}), 7.86$ (d, $J=9.2$ $\mathrm{Hz}, 2 \mathrm{H}, \mathrm{ArH}), 7.90$ (d, $J=8.5 \mathrm{~Hz}, 2 \mathrm{H}, \mathrm{ArH}), 8.04$ (dd, $J=7.9$ and $2.1 \mathrm{~Hz}, 1 \mathrm{H}, \mathrm{PyH}), 8.47-8.50$ (m, 3H, PyH), $8.88(\mathrm{~d}, J=2.1 \mathrm{~Hz}, 1 \mathrm{H}, \mathrm{PyH}) .{ }^{13} \mathrm{C}$ NMR $\left(125 \mathrm{MHz}, \mathrm{CDCl}_{3}\right): \delta=28.7,28.8,38.9,39.0,40.2\left(\mathrm{CH}_{3}\right), 59.0,60.7$ (PyC), 60.8 (РyC), 67.2 (COH), 70.10, 70.15, 70.3, 70.5, 70.6, 111.4, 121.96, 122.00, 122.04, 122.6, 123.0, 125.3, 127.9, 128.7, 134.9, 135.5, 136.4, 143.6, 147.3, 148.89, 148.93, 152.7, 154.8, 159.2, 159.3, 162.5, 165.3 (CO), 166.8 (CO).

\subsection{Fluorescence Analysis}

All fluorescence measurements were performed in triplicate or more by using an aqueous solution of $10 \mathrm{mM}$ Hepes- $\mathrm{NaOH}(\mathrm{pH}$ 7.4) containing an appropriate concentration of FMN and $0.10 \mathrm{M} \mathrm{NaCl}$ in the absence or presence of the Dabcyl-labeled Phos-tag ligand (micromolar concentrations) and more than two equivalents of $\mathrm{ZnCl}_{2}$. The sample solution (3 ml) was continuously stirred at $25.0^{\circ} \mathrm{C} \pm 0.1^{\circ} \mathrm{C}$. Under the experimental conditions, the Phos-tag ligand coordinated almost quantitatively with two equivalents of zinc(II) ion to form its dizinc(II) complex [8] [12]; this is abbreviated as Dabcyl-Phos-tag throughout this article (for structure, see Figure 1). 


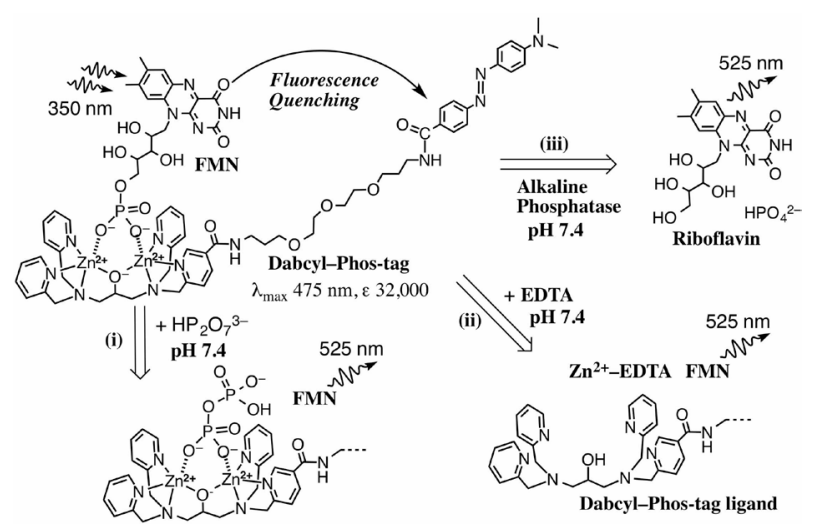

Figure 1. Structure of FMN-bound Dabcyl-Phos-tag complex and three methods for releasing the FMN molecule to reduce the fluorescence quenching effect at $\mathrm{pH} 7.4$ in aqueous solution: (i) Replacing the FMN molecule by a pyrophosphate anion to form pyrophosphate-bound Dabcyl-Phos-tag; (ii) Demetalation of the dinuclear zinc(II) complex by incubation with EDTA; (iii) Dephosphorylation of FMN by AP to produce riboflavin and inorganic phosphate.

\section{Results and Discussion}

\subsection{Characteristics of Dabcyl-Phos-Tag}

Dabcyl-labeled Phos-tag ligand containing a hydrophilic ether linker was synthesized by a coupling reaction of an amino-pendent Phos-tag derivative [12] and an active derivative of Dabcyl acid in 84\% yield (see Materials and methods). The solubility of Dabcyl-labeled Phos-tag ligand in an aqueous solution (pH 7.4, $10 \mathrm{mM}$ Hepes$\mathrm{NaOH}, 0.10 \mathrm{M} \mathrm{NaCl}$ ) was determined to be $15 \mu \mathrm{M}$ at $25^{\circ} \mathrm{C}$. The addition of $1.0 \mathrm{mM} \mathrm{ZnCl}_{2}$ to form the dizinc(II) complex of the Phos-tag ligand resulted in an increase in the solubility to $92 \mu \mathrm{M}$ in the same buffer solution; this was sufficient to permit fluorescence analysis without the need for an organic solvent such as ethanol. The structure of the dinuclear zinc(II) complex (Dabcyl-Phos-tag) is shown in Figure 1. The UV-visible absorption spectrum of Dabcyl-Phos-tag $(15 \mu \mathrm{M})$ in a buffer solution (pH 7.4) containing $10 \mathrm{mM}$ Hepes-NaOH, $0.10 \mathrm{M} \mathrm{NaCl}$, and $50 \mu \mathrm{M} \mathrm{ZnCl}_{2}$ at $25^{\circ} \mathrm{C}$ is shown in Figure 2(a). The absorption maximum $\left(\lambda_{\max }\right)$ is $475 \mathrm{~nm}$ with $\varepsilon=3.2 \times 10^{4}$ $\mathrm{M}^{-1} \cdot \mathrm{cm}^{-1}$ and the absorption valley is around $350 \mathrm{~nm}$. The absorption and emission spectra of $15 \mu \mathrm{M}$ FMN in the same buffer solution are shown in Figure 2(b) and Figure 2(c), respectively. The visible absorption intensity of $\operatorname{FMN}\left(\lambda_{\text {peak } 1}=373 \mathrm{~nm}, \varepsilon=1.05 \times 10^{4} \mathrm{M}^{-1} \cdot \mathrm{cm}^{-1} ; \lambda_{\text {peak } 2}=445 \mathrm{~nm}, \varepsilon=1.25 \times 10^{4} \mathrm{M}^{-1} \cdot \mathrm{cm}^{-1}\right.$ [18] $)$ is much smaller than that of Dabcyl-Phos-tag, whereas the UV absorption intensity of FMN at around $350 \mathrm{~nm}$ is stronger than that of Dabcyl-Phos-tag. The fluorescence spectrum $\left(\lambda_{\mathrm{em}}=525 \mathrm{~nm}\right)$ of FMN therefore shows a considerable overlap with the strong visible absorption band of Dabcyl-Phos-tag. These spectrophotometric data led us to develop a novel application of the phosphate-binding quencher, Dabcyl-Phos-tag and the fluorogenic AP-substrate, FMN.

\subsection{Analysis of the Phos-Tag-Based Fluorescence Quenching System}

We conducted the quenching analysis by using Dabcyl-Phos-tag and FMN in an aqueous solution (pH 7.4; 10 $\mathrm{mM}$ Hepes- $\mathrm{NaOH}, 0.10 \mathrm{M} \mathrm{NaCl})$ at $25^{\circ} \mathrm{C}$. The test solution $(3.0 \mathrm{ml})$ contained $13.5 \mu \mathrm{M}$ Dabcyl-labeled Phostag ligand, $0.10 \mathrm{mM} \mathrm{ZnCl}_{2}$, and an appropriate concentration of FMN (0 - $\left.32 \mu \mathrm{M}\right)$. Reference experiments were performed with the addition of EDTA $(2.0 \mathrm{mM})$ as a strong chelator of zinc(II) that can remove the zinc(II) ion from the Phos-tag molecule [12]. The fluorescence intensities (FI, arbitrary unit) at $530 \mathrm{~nm}$ with excitation at $350 \mathrm{~nm}$ are shown in Figure 3. For the zinc(II)-bound condition (Figure 3(a)), obvious quenching was observed up to one equivalent of FMN $(13.5 \mu \mathrm{M})$; further addition of FMN $(\geq 16 \mu \mathrm{M})$ resulted in a dose-dependent increase in the fluorescence intensity. Therefore, the fluorescence quenching is stoichiometric and results from the formation of a 1:1 complex between Dabcyl-Phos-tag and FMN under the experimental conditions.

No such quenching was observed in a reference experiment conducted in the presence of an excess of EDTA (Figure 3(b)), where the fluorescence intensity increased linearly with increasing concentration of FMN (0 - 20 


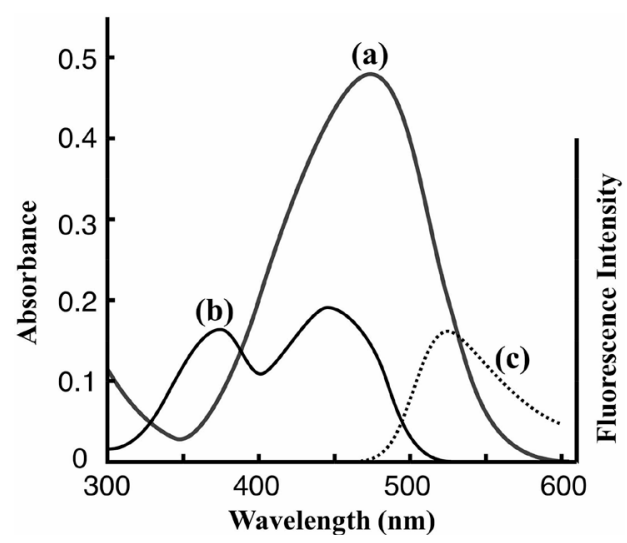

Figure 2. Absorption spectra at $25^{\circ} \mathrm{C}$ and $\mathrm{pH} 7.4$ (10 mM Hepes- $\mathrm{NaOH}$, $0.10 \mathrm{M} \mathrm{NaCl}$ ): (a) Mixture of $15 \mu \mathrm{M}$ Dabcyl-labeled Phos-tag ligand and $50 \mu \mathrm{M} \mathrm{ZnCl}, \lambda_{\max }=475 \mathrm{~nm}\left(\varepsilon=32,000 \mathrm{M}^{-1} \cdot \mathrm{cm}^{-1}\right)$; (b) $15 \mu \mathrm{M}$ FMN, $\lambda_{\text {peak } 1}=373 \mathrm{~nm}\left(\varepsilon=10,500 \mathrm{M}^{-1} \cdot \mathrm{cm}^{-1}\right), \lambda_{\text {peak } 2}=445 \mathrm{~nm}(\varepsilon=$ $12,500 \mathrm{M}^{-1} \cdot \mathrm{cm}^{-1}$ ); (c) Fluorescence spectrum of $15 \mu \mathrm{M}$ FMN with excitation at $350 \mathrm{~nm}$ at $25^{\circ} \mathrm{C}$ and $\mathrm{pH} 7.4(10 \mathrm{mM}$ Hepes-NaOH, 0.10 M $\mathrm{NaCl}): \lambda_{\mathrm{em}}=525 \mathrm{~nm}$.

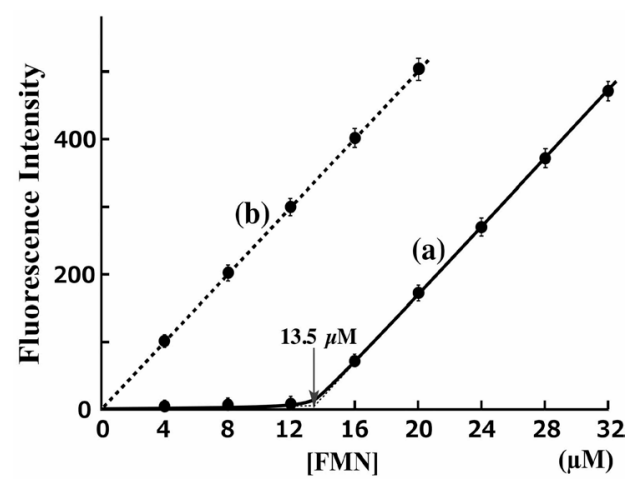

Figure 3. Fluorescence emission response at various concentrations of FMN in the presence of $13.5 \mu \mathrm{M}$ Dabcyl-labeled Phos-tag ligand and $0.10 \mathrm{mM} \mathrm{ZnCl}_{2}$ : (a) With no additive and (b) in the presence of $2.0 \mathrm{mM}$ EDTA. Fluorescence intensities at $530 \mathrm{~nm}$ with excitation at $350 \mathrm{~nm}$ at $25^{\circ} \mathrm{C}$ and $\mathrm{pH} 7.4$ (10 mM Hepes- $\left.\mathrm{NaOH}, 0.10 \mathrm{M} \mathrm{NaCl}\right)$.

$\mu \mathrm{M})$. The slope of the fluorescence intensity in Figure 3(b) is almost the same as that in Figure 3(a) over the FMN concentration range 16 - $32 \mu \mathrm{M}$. The quenching efficiency of a mixture of $13.5 \mu \mathrm{M}$ Dabcyl-Phos-tag and $12 \mu \mathrm{M}$ FMN was more than 95\% in comparison to the fluorescence intensity of the reference experiment using the same concentration of FMN. Furthermore, a mixture of the dephosphorylated counterpart, riboflavin (1 - 10 $\mu \mathrm{M}$ ), and 13.5 $\mu \mathrm{M}$ Dabcyl-Phos-tag showed no quenching under the same experimental conditions. The donor-to-acceptor distance for an efficient fluorescence quenching system should be in a range 1 to $10 \mathrm{~nm}$ [23]. The distance between the flavin moiety and the Dabcyl group in the 1:1 complex of FMN and Dabcyl-Phos-tag is less than $3 \mathrm{~nm}$, as shown by the general molecular model (see Figure 1). These results clearly demonstrate that efficient quenching of FMN is caused by the coordination of phosphate to the zinc(II) ions of Dabcyl-Phos-tag. From the fluorescence intensities of equimolar amounts $(13.5 \mu \mathrm{M})$ of FMN and Dabcyl-Phos-tag in the absence $(F I=30)$ and the presence $(F I=340)$ of EDTA, and the base-line $F I$ value of 10 , extrapolated from the fluorescence-response curve (see Figure 3(a)), the dissociation constant $K_{\mathrm{d}}(\mu \mathrm{M})$ of the 1:1 complex of FMN-bound Dabcyl-Phos-tag $\left(K_{\mathrm{d}}=\right.$ [FMN][Dabcyl-Phos-tag]/[1:1 complex] $)$ is estimated to be less than 0.1 $\mu \mathrm{M}$ under the experimental conditions: A predicted quenching constant (QC) is $0.97(=330 / 340)$; concentrations of [1:1 complex] and [FMN] (= [Dabcyl-Phos-tag]) could be calculated, respectively, to be (310/QC)/340 × 13.5 $\mu \mathrm{M}$ and $[1-(310 / \mathrm{QC}) / 340] \times 13.5 \mu \mathrm{M}$. 


\subsection{Methods for Releasing FMN from Its Dabcyl-Phos-Tag Complex}

We recently reported that the rapid equilibrium involved in the binding of a phosphorylated peptide to a fluorogenic Phos-tag molecule can be applied in real-time analyses of phosphatase and kinas profiles on a time scale of minutes [13] [14]. If the change in the binding level of FMN to Dabcyl-Phos-tag is as fast as that for the previous Phos-tag system, the fluorescence quenching system might be suitable for a novel real-time analytical method that uses as an index the amount of Phos-tag-bound FMN. In relation to the dissociation kinetics of the Dabcyl-Phos-tag complex with FMN, there are three possible routes for the release of FMN, as shown in Figure 1. In route (i), the FMN molecule is replaced by another phosphate moiety, such as an inorganic pyrophosphate, to form a pyrophosphate-bound Dabcyl-Phos-tag molecule. In route (ii), the zinc(II) ions of the Dabcyl-Phostag are removed by incubation with a strong chelating reagent such as EDTA to give the metal-free Phos-tag ligand. In route (iii), the 5'-phosphate group of FMN is eliminated by a phosphatase to form riboflavin and an inorganic phosphate.

First, we examined the rate of the phosphate-exchange reaction for the FMN-bound Dabcyl-Phos-tag in the presence of large excesses of sodium pyrophosphate at $25^{\circ} \mathrm{C}$ [route (i) in Figure 1]. A sample solution (3.0 ml) containing $14 \mu \mathrm{M}$ FMN, $13.5 \mu \mathrm{M}$ Dabcyl-Phos-tag (13.5 $\mu \mathrm{M}$ ligand, $50 \mu \mathrm{M} \mathrm{ZnCl}_{2}$ ), $0.10 \mathrm{M} \mathrm{NaCl}$, and $10 \mathrm{mM}$ Hepes-NaOH (pH 7.4) was prepared and loaded into the fluorescence spectrophotometer with stirring. Immediately after injection of $0.10 \mathrm{M}$ aqueous sodium pyrophosphate (pH 7.4, $0.16 \mathrm{ml}$; final concentration $5.1 \mathrm{mM}$ ) to the sample solution, the fluorescence at $530 \mathrm{~nm}$ was measured for $5 \mathrm{~min}$. The fluorescence intensity initially increased rapidly, reaching a plateau within $2 \mathrm{~s}$, the final value of which was equal to that for $100 \%$ release of FMN in bulk solution. The rapid increase in fluorescence showed that the phosphate-exchange reaction occurs on a time scale of seconds under the experimental conditions.

To study route (ii), we conducted the same kinetic experiment except that a $0.10 \mathrm{M}$ aqueous solution of

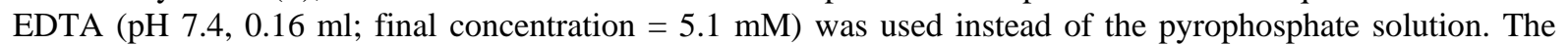
fluorescence intensity once more increased gradually as a result of demetalation by the EDTA. The time for $50 \%$ release of FMN was $32 \mathrm{~s}$, which was much slower than that for the pyrophosphate-exchange reaction in route (i). Subsequently, the fluorescence intensity increased gradually and reached the same plateau value as that for route (i), showing the zinc(II)-chelating ability of EDTA is much stronger than that of the Phos-tag ligand under the experimental conditions. The rates of phosphate release by routes (i) and (ii) should be useful for optimizing an experimental protocol to recover an intact phosphoric monoester from its Phos-tag complex. In the next section, we describe route (iii) through AP reaction.

\subsection{Real-Time Analysis of FMN Dephosphorylation by AP}

Next, we used our Phos-tag-based fluorescence quenching system to study the kinetics of the dephosphorylation of FMN by an AP [see route (iii) in Figure 1]. A real-time analysis of the dephosphorylation of FMN was performed by using a 1-cm quartz cell with stirring at $25^{\circ} \mathrm{C}$ for $20 \mathrm{~min}$. The reaction mixture (3 ml) contained 3.0 $\mu \mathrm{M}$ FMN, $4.5 \mu \mathrm{M}$ Dabcyl-Phos-tag ( $4.5 \mu \mathrm{M}$ ligand and $30 \mu \mathrm{M} \mathrm{ZnCl}$ ), $0.10 \mathrm{M} \mathrm{NaCl}, 1.0 \mathrm{mM} \mathrm{MgCl}$, and 10 $\mathrm{mM}$ Hepes- $\mathrm{NaOH}$ ( $\mathrm{pH}$ 7.4). The dephosphorylation reaction was initiated by the injection of an appropriate amount of bovine intestinal mucosa AP. Immediately, the fluorescence intensity at $530 \mathrm{~nm}$ increased in a time-dependent manner and was subsequently measured at $10 \mathrm{~s}$ interval over $20 \mathrm{~min}$. The change in sample volume as a result of the injection of the enzyme solution was less than $1 \%$. The rate of the dephosphorylation increased with increasing concentration of the AP. Typical fluorescence changes in the presence of four concentrations of the AP $(0.23,0.45,0.91$, and 1.8 units $/ \mathrm{ml})$ are shown in Figure 4. HPLC analysis confirmed that riboflavin (dephosphorylated FMN) was the final product formed by treatment with the AP, and that no other flavin derivatives were present. The enhancement in fluorescence resulting from the elimination of the 5'-phosphate group from FMN was more than 18-fold. The dephosphorylation reaction progressed according to pseudo-first-order kinetics until the fluorescence intensity reached about half the final plateau value. The initial rates of the dephosphorylation were almost proportional to the amount of AP. From the change in the time course of the fluorescence intensity in the presence of 0.91 units $/ \mathrm{ml}$ of AP, the half-life times of the dephosphorylation reaction was estimated to be 4 min. Furthermore, no fluorescence enhancement was observed in a reference experiment in the absence of the AP under the same conditions (diamonds; Figure 4). The fluorescence change is therefore consistent with time-dependent dephosphorylation of FMN to produce stoichiometric amounts of inorganic phosphate and riboflavin. Consequently, this fluorescence quenching system can be used to assay the activity of APs under near-physiological conditions. 


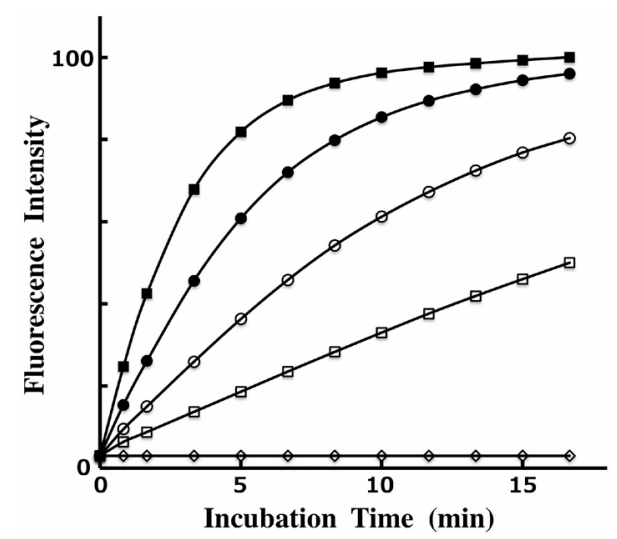

Figure 4. Time course changes of the fluorescence intensity of an aqueous solution ( $\mathrm{pH}$ 7.4) containing $3.0 \mu \mathrm{M}$ FMN, $4.5 \mu \mathrm{M}$ DabcylPhos-tag (4.5 $\mu \mathrm{M}$ Phos-tag ligand, $30 \mu \mathrm{M} \mathrm{ZnCl}$ ), $0.10 \mathrm{M} \mathrm{NaCl}, 1.0$ $\mathrm{mM} \mathrm{MgCl}_{2}$, and $10 \mathrm{mM}$ Hepes- $\mathrm{NaOH}$ at $530 \mathrm{~nm}$ with excitation at 350 $\mathrm{nm}$ at $25^{\circ} \mathrm{C}$ in the absence or presence of bovine intestinal mucosa AP: 0 units/ml (diamonds), 0.23 units/ml (open squares), 0.45 units $/ \mathrm{ml}$ (open circles), 0.91 units/ml (black circles), and 1.8 units/ml (black squares).

\subsection{Phosphatase Inhibition Profiling by Using the Dabcyl-Phos-Tag Quenching System}

To test the possibility of using the Phos-tag-based fluorescence quenching system as a novel screening method for the discovery of AP inhibitors, we examined the inhibition of bovine intestinal mucosa AP ( 0.18 units/ml) by two typical inhibitors (vanadate and levamisole) in a similar real-time procedure to that described in the previous section. Inhibition assays were performed at $\mathrm{pH} 7.4$ in the presence of vanadate ion as a competitive inhibitor or a levamisole (monoprotonated form) as a noncompetitive inhibitor. The time-course changes in the fluorescence intensity at $530 \mathrm{~nm}$ were monitored continuously until $10 \%$ of the FMN was hydrolyzed. The residual activity ratios (\%) were evaluated from the initial rates of the FMN-dephosphorylation reaction in the absence and presence of the inhibitor. The dephosphorylation rate decreased in a dose-dependent manner with vanadate anion. The inhibition curve shown in Figure 5(A) (open squares) is a plot of residual phosphatase activity against the concentration of the inhibitor on a logarithmic scale. From the resulting sigmoidal inhibition curve, we evaluated the half maximal inhibitory concentration $\left(I C_{50}\right)$ of vanadate to be $0.12 \mu \mathrm{M}$ at $\mathrm{pH}$ 7.4. The reported $I C_{50}$ values for vanadate anion in vitro are $0.5 \mu \mathrm{M}$ for an AP from human small-intestinal mucosa at $\mathrm{pH} 9.0$ [20] and $2.5 \mu \mathrm{M}$ for an AP from Escherichia coli at $\mathrm{pH} 8.0$ [19], both determined by means of a spectrophotometric kinetic assay with 4-nitrophenyl phosphate as a substrate. Differences in the $I C_{50}$ values appeared to reflect the use of different isoenzymes and/or substrates and differences in components such as the buffer or additional salt. For levamisole, no inhibition of the AP from bovine intestinal mucosa was observed up to a concentration of 1 $\mathrm{mM}$. The organ specificity of levamisole had been previously examined for various human AP isoforms. The study showed that AP isoforms from the liver, kidney, and spleen were more strongly inhibited $\left(I_{50}=\sim 10 \mu \mathrm{M}\right)$, whereas those from the intestine and placenta were much more weakly inhibited $\left(I C_{50}>1 \mathrm{mM}\right)$ [21]. We therefore conducted a similar inhibition experiment with another AP from bovine kidney. Figure 5(B) shows the resulting inhibition profile with 0.16 units/ml of the kidney isoform of AP in the presence of various concentrations of levamisole. A similar dose-dependent inhibition, with an $I C_{50}$ value of $22 \mu \mathrm{M}$, was observed. In addition, we evaluated the $I C_{50}$ value of the kidney isoform for vanadate anion to be $1.0 \mu \mathrm{M}$ (open circles in Figure 5(A)), a weaker inhibition effect than that for the intestinal isoform (open squares in Figure 5(A)). Therefore, the Dabcyl-Phos-tag quenching system using FMN, which can act as a substrate for AP, might be useful in a simple preliminary screening procedure for novel inhibitors of APs in aqueous solution at $\mathrm{pH}$ 7.4.

\section{Conclusions}

We have developed a novel application of the Phos-tag-based fluorescence quenching system in the analysis of the activity of AP in the dephosphorylation of FMN under conditions that are as close as possible to physiological ones. For this system, we synthesized Dabcyl-Phos-tag, consisting of a phosphate-binding tag molecule 

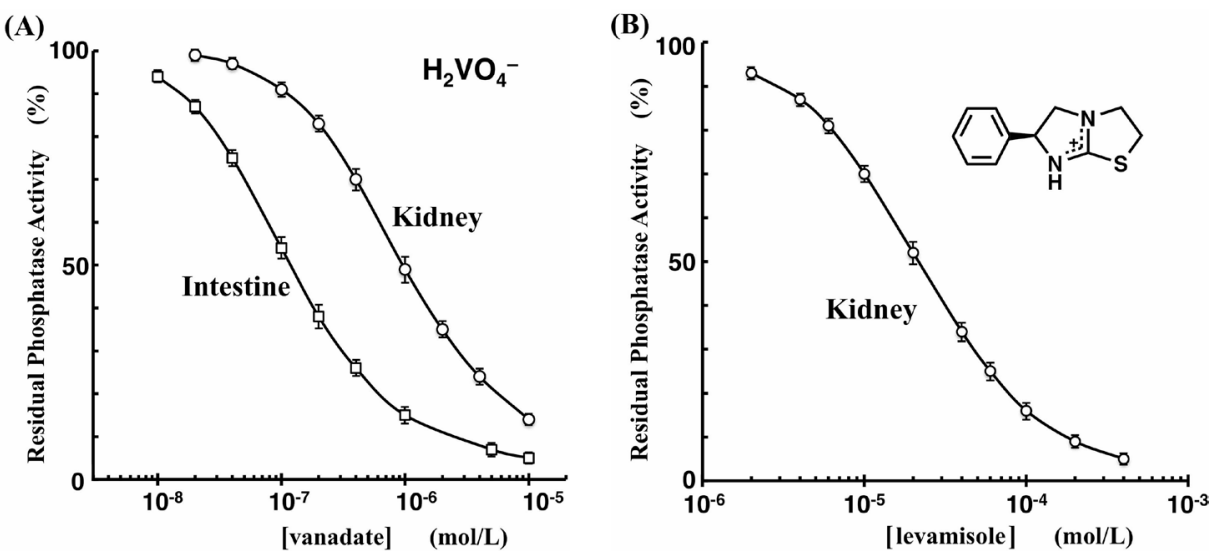

Figure 5. Inhibition curves of the AP reactions in the presence of phosphatase inhibitor ((A) vanadate, (B) levamisole) at $25^{\circ} \mathrm{C}$. The ordinates represent the residual phosphatase activity ratio (\%). The reaction mixture (pH 7.4, $3.0 \mathrm{ml}$ ) contained 3.0 $\mu \mathrm{M}$ FMN, $4.5 \mu \mathrm{M}$ Dabcyl-Phos-tag (4.5 $\mu \mathrm{M}$ Phos-tag ligand, $30 \mu \mathrm{M} \mathrm{ZnCl}_{2}$ ), $0.10 \mathrm{M} \mathrm{NaCl}, 1.0 \mathrm{mM} \mathrm{MgCl}$, and $10 \mathrm{mM}$ Hepes- $\mathrm{NaOH}$. The amount of bovine AP was $0.18 \mathrm{units} / \mathrm{ml}$ of intestinal mucosa isoform (open squares) or 0.16 units $/ \mathrm{ml}$ of bovine kidney isoform (open circles).

(Phos-tag) attached to a Dabcyl group. The Phos-tag derivative preferentially captured FMN, resulting in efficient quenching of the fluorescence of the flavin moiety. This quenching system was used in continuous monitoring of the phosphorylation status of FMN in the presence of AP. In addition, inhibition assays were used to assess the $I C_{50}$ values of vanadate and levamisole in phosphatase reactions for two kinds of AP isoenzyme. The quenching system has the following significant advantages.

a) The analysis is a relatively simple procedure involving three aqueous solutions of Dabcyl-Phos-tag, FMN, and AP, respectively.

b) It is not necessary to label the AP substrate with a radioactive isotope of phosphorus or to use an artificial fluorophore.

c) Real-time analysis of the FMN-dephosphorylation reaction is possible without multiple sampling.

d) The total analysis time is less than 15 minutes.

e) Finally, the sample volume can be reduced to sub-milliliter quantities.

Thus, the principle of this Phos-tag-based fluorescence quenching system can be applied in simple and reliable profiling of AP to identify inhibitors (or activators) or to analyze their activities and requires only a standard laboratory fluorescence spectrometer.

\section{Acknowledgements}

This work was supported in part by KAKENHI Grants (24590050, 25293005, 25560417, 25117718, and 26460036) from the Japan Society for the Promotion of Science (JSPS) and a research grant from the Takeda Science Foundation.

\section{References}

[1] Coleman, J.E. (1992) Structure and Mechanism of Alkaline Phosphatase. Annual Review of Biophysics and Biomolecular Structure, 21, 441-483. http://dx.doi.org/10.1146/annurev.bb.21.060192.002301

[2] Millán, J.L. (2006) Alkaline Phosphatases: Structure, Substrate Specificity and Functional Relatedness to Other Members of a Large Superfamily of Enzymes. Purinergic Signaling, 2, 335-341. http://dx.doi.org/10.1007/s11302-005-5435-6

[3] Stinghen, S.T., Moura, J.F., Zancanella, P., Rodrigues, G.A., Pianovski, M.A., Lalli, E., Arnold, D.L., Minozzo, J.C., Callefe, L.G., Ribeiro, R.C. and Figueiredo, B.C. (2006) Specific Immunoassays for Placental Alkaline Phosphatase as a Tumor Marker. Journal of Biomedicine and Biotechnology, 2006, Article ID: 56087. http://dx.doi.org/10.1155/JBB/2006/56087

[4] Peake, M.J., Pejakovic, M. and White, G.H. (1988) Quantitative Method for Determining Serum Alkaline Phosphatase Isoenzyme Activity: Estimation of Intestinal Component. Journal of Clinical Pathology, 41, 202-206. http://dx.doi.org/10.1136/jcp.41.2.202

[5] Schrenkhammer, P., Rosnizeck, I.C., Duerkop, A., Wolfbeis, O.S. and Schäferling, M. (2008) Time-Resolved Fluores- 
cence-Based Assay for the Determination of Alkaline Phosphatase Activity and Application to the Screening of Its Inhibitors. Journal of Biomolecular Screening, 13, 9-16. http://dx.doi.org/10.1177/1087057107312031

[6] Liu, Y. and Schanze, K.S. (2008) Conjugated Polyelectrolyte-Based Real-Time Fluorescence Assay for Alkaline Phosphatase with Pyrophosphate as Substrate. Analytical Chemistry, 80, 8605-8612. http://dx.doi.org/10.1021/ac801508y

[7] Gu, X., Zhang, G., Wang, Z., Liu, W., Xiao, L. and Zhang, D. (2013) A New Fluorometric Turn-On Assay for Alkaline Phosphatase and Inhibitor Screening Based on Aggregation and Deaggregation of Tetraphenylethylene Molecules. Analyst, 138, 2427-2431. http://dx.doi.org/10.1039/c3an36784c

[8] Kinoshita, E., Takahashi, M., Takeda, H., Shiro, M. and Koike, T. (2004) Recognition of Phosphate Monoester Dianion by an Alkoxide-Bridged Dinuclear Zinc(II) Complex. Dalton Transactions, 1189-1193. http://dx.doi.org/10.1039/b400269e

[9] Kinoshita, E., Kinoshita-Kikuta, E., Takiyama, K. and Koike, T. (2006) Phosphate-Binding Tag, a New Tool to Visualize Phosphorylated Proteins. Molecular and Cellular Proteomics, 5, 749-757. http://dx.doi.org/10.1074/mcp.T500024-MCP200

[10] Kinoshita-Kikuta, E., Aoki, Y., Kinoshita, E. and Koike, T. (2007) Label-Free Kinase Profiling Using Phosphate Affinity Polyacrylamide Gel Electrophoresis. Molecular and Cellular Proteomics, 6, 356-366. http://dx.doi.org/10.1074/mcp.T600044-MCP200

[11] Kinoshita, E. and Kinoshita-Kikuta, E. (2011) Improved Phos-Tag SDS-PAGE under Neutral pH Conditions for Advanced Protein Phosphorylation Profiling. Proteomics, 11, 319-323. http://dx.doi.org/10.1002/pmic.201000472

[12] Tsunehiro, M., Meki, Y., Matsuoka, K., Kinoshita-Kikuta, E., Kinoshita, E. and Koike, T. (2013) A Phos-Tag-Based Magnetic-Bead Method for Rapid and Selective Separation of Phosphorylated Biomolecules. Journal of Chromatography B, 925, 86-94. http://dx.doi.org/10.1016/j.jchromb.2013.02.039

[13] Takiyama, K., Kinoshita, E., Kinoshita-Kikuta, E., Fujioka, Y., Kubo, Y. and Koike T. (2009) A Phos-Tag-Based Fluorescence Resonance Energy Transfer System for the Analysis of the Dephosphorylation of Phosphopeptides. Analytical Biochemistry, 388, 235-241. http://dx.doi.org/10.1016/j.ab.2009.02.039

[14] Somura, M., Takiyama, K., Kinoshita-Kikuta, E., Kinoshita, E. and Koike, T. (2011) A Phos-Tag-Based Fluorescence Energy Transfer System for the Analysis of the Kinase Reaction of a Substrate Peptide. Analytical Methods, 3, 13031309. http://dx.doi.org/10.1039/C1AY05016H

[15] Sapsford, K.E., Berti, L. and Medintz, I.L. (2006) Materials for Fluorescence Resonance Energy Transfer Analysis: Beyond Traditional Donor-Acceptor Combinations. Angewandte Chemie International Edition, 45, 4562-4588. http://dx.doi.org/10.1002/anie.200503873

[16] Kolpashchikov, D.M. (2012) An Elegant Biosensor Molecular Beacon Probe: Challenges and Recent Solutions. Scientifica, 2012, Article ID: 928783. http://dx.doi.org/10.6064/2012/928783

[17] Johansson, M.K. (2006) Choosing Reporter-Quencher Pairs for Efficient Quenching through Formation of Intramolecular Dimers. Methods in Molecular Biology, 335, 17-29. http://dx.doi.org/10.1385/1-59745-069-3:17

[18] Nielsen, P., Rauschenbach, P. and Bacher, A. (1983) Phosphates of Riboflavin and Riboflavin Analogs: A Reinvestigation by High-Performance Liquid Chromatography. Analytical Biochemistry, 130, 359-368. http://dx.doi.org/10.1016/0003-2697(83)90600-0

[19] Lopez, V., Stevens, T. and Lindquist, R.N. (1976) Vanadium Ion Inhibition of Alkaline Phosphatase-Catalyzed Phosphate Ester Hydrolysis. Archives of Biochemistry and Biophysics, 175, 31-38. http://dx.doi.org/10.1016/0003-9861(76)90482-3

[20] Seargeant, L.E. and Stinson, R.A. (1979) Inhibition of Human Alkaline Phosphatase by Vanadate. Biochemical Journal, 181, 247-250. http://www.ncbi.nlm.nih.gov/pmc/articles/PMC1161148/

[21] Van Belle, H. (1976) Alkaline Phosphatase. I. Kinetics and Inhibition by Levamisole of Purified Isoenzymes from Humans. Clinical Chemistry, 22, 972-976. http://www.clinchem.org/content/22/7/972.abstract

[22] Kozlenkov, A., Le Du, M.H., Cuniasse, P., Ny, T., Hoylaerts, M.F. and Millán, J.L. (2004) Residues Determining the Binding Specificity of Uncompetitive Inhibitors to Tissue-Nonspecific Alkaline Phosphatase. Journal of Bone and Mineral Research, 19, 1862-1871. http://dx.doi.org/10.1359/JBMR.040608

[23] Wu, P. and Brand, L. (1994) Resonance Energy Transfer: Methods and Applications. Analytical Biochemistry, 218, 113. http://dx.doi.org/10.1006/abio.1994.1134 
Scientific Research Publishing (SCIRP) is one of the largest Open Access journal publishers. It is currently publishing more than 200 open access, online, peer-reviewed journals covering a wide range of academic disciplines. SCIRP serves the worldwide academic communities and contributes to the progress and application of science with its publication.

Other selected journals from SCIRP are listed as below. Submit your manuscript to us via either submit@scirp.org or Online Submission Portal.
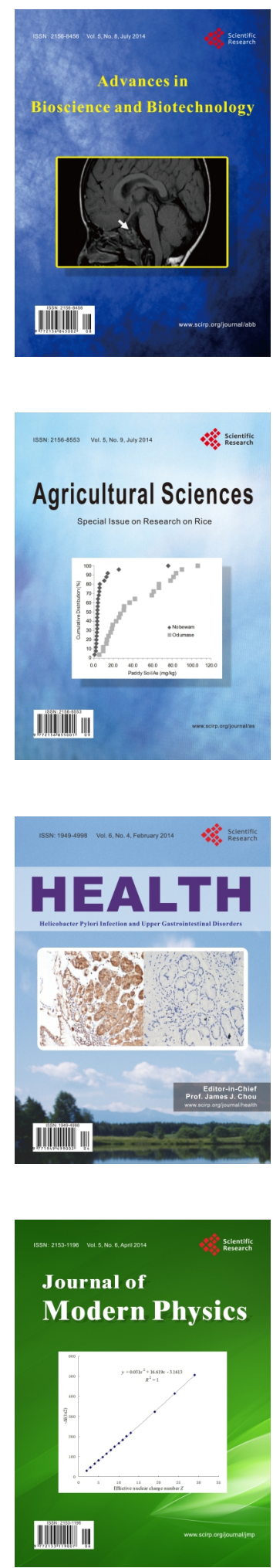
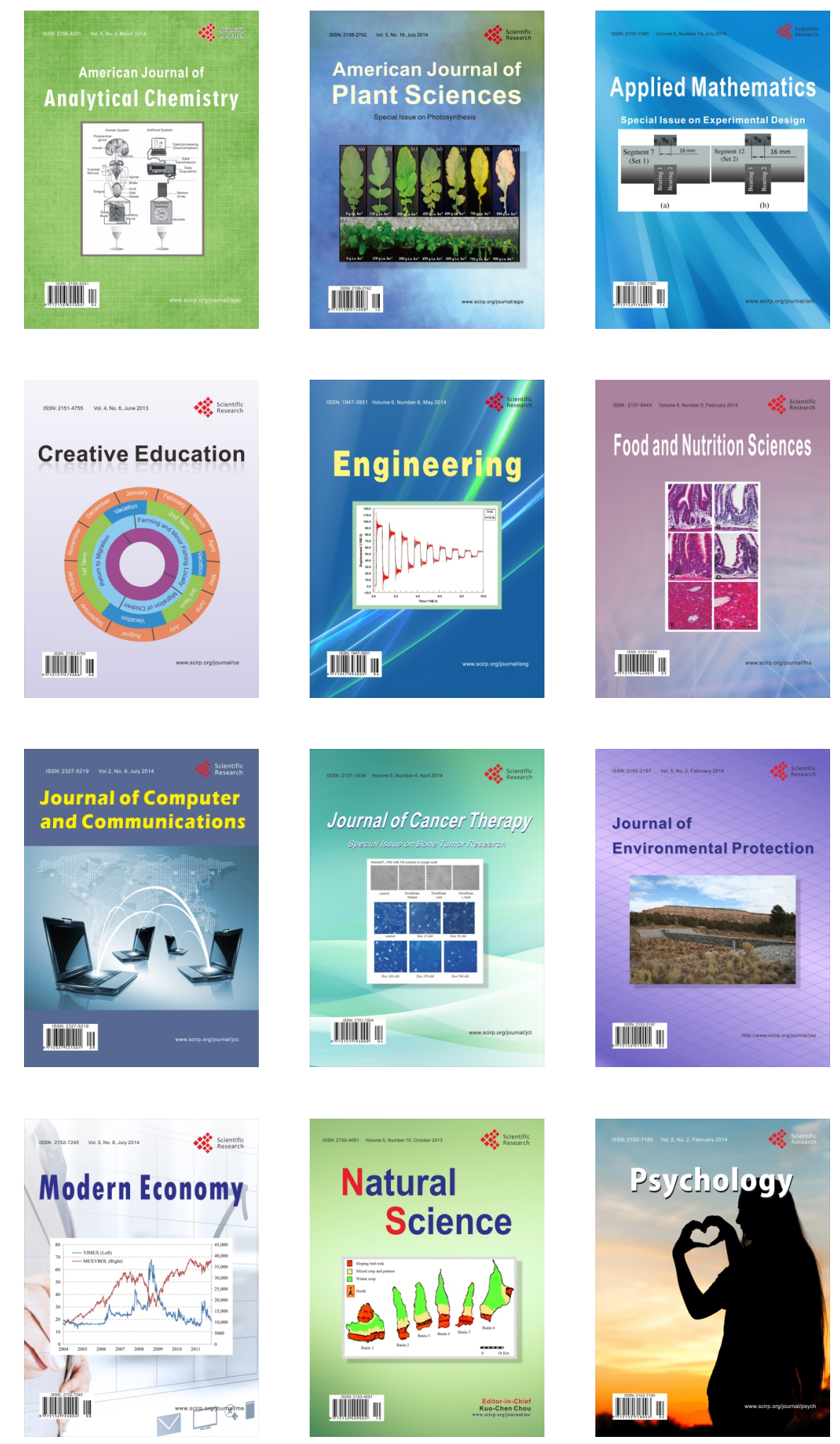